\title{
Analysis of Methodological Quality of The Process of Moving From Evidence to Recommendations in Chinese Clinical Practice Guidelines Between 2010 and 2020: A Cross-Sectional Study
}

\author{
Nan Zheng \\ Qilu Hospital of Shandong University \\ Xiaoming Chen \\ Qilu Hospital of Shandong University \\ Xiangying Ren \\ College of Nursing and Health, Henan University \\ Xiangyun Guan \\ Qilu Hospital of Shandong University \\ Ran Tan \\ Qilu Hospital of Shandong University \\ Linxia Su \\ Qilu Hospital of Shandong University \\ Qiao Huang \\ Zhongnan Hospital of Wuhan University, Wuhan University \\ Yingjuan Cao \\ Qilu Hospital of Shandong University \\ Yinghui Jin ( $\nabla$ jinyinghuiebm@163.com ) \\ Zhongnan Hospital of Wuhan University, Wuhan University
}

\section{Research Article}

Keywords: Chinese clinical practice guidelines, evidence to recommendations, methodology quality, methodological characteristics.

Posted Date: February 10th, 2022

DOI: https://doi.org/10.21203/rs.3.rs-1255887/v1

License: (c) (1) This work is licensed under a Creative Commons Attribution 4.0 International License. Read Full License 


\section{Abstract}

Background: The general situation of the methodological quality of the process of moving from evidence to recommendations (EtR) in Chinese CPGs and the main methodological factors affecting the rigor of EtR is still unclear. This study explored the status of the methodological characteristics of EtR by analyzing of all Chinese CPGs illustrating recognizable recommendations published during the period 2010-2020, and identified the relevant factors associated with the rigor of EtR in CPGs.

Methods: We systematically searched two major Chinese databases, CNKI and WanFang Database to gather all CPGs illustrating recognizable recommendations from 2010 to 2020 . A data-extraction form was designed to extract all the methodological characteristics of EtR. We used the M-K test to identify monotonically increasing or decreasing trends of each methodological characteristic over time, and compared the methodological characteristics between groups using Chi-square test. Multi-factor Binary Logistic regression analysis was used to analyze factors related to the rigor of the process of EtR in CPGs.

Results: A total of 753 CPGs (EB-CPGs=386/1,127, CB-CPGs=367/1,527) were selected based on the selection criteria. EB-CPGs did better than CB-CPGs in most of the EtR methodological characteristics, and CPGs using GRADE were also superior to those not using GRADE. 43.69\% (329/753) of CPGs considered relevant factors. The most frequently considered factors were the effectiveness of the intervention $(398,52.9 \%)$, safety $(368,48.9 \%)$, and balance of benefits and harms $(193,25.6 \%)$. Only 95 CPGs considered costs in the methods or the process of EtR, the proportion was even lower for the consideration of values and preferences $(57,7.6 \%)$ and feasibility $(47,6.2 \%)$. The rigor of EtR were significantly associated with GRADE or non-GRADE CPGs (OR 2.03, 95\% Cl 1.37-3.00, $P<0.001$ ), EB-CPGs or CB-CPGs (OR 1.48, 95\% Cl 1.06-2.07, $P=0.022)$ and the designated level of evidence (OR $1.64,95 \% \mathrm{Cl}$ $1.15-2.34, P=0.006)$.

Conclusion: Unsatisfactory: there is still much room for improvement in the rigor of EtR in Chinese CPGs over the last 11 years. In order to improve the rigor of EtR, guideline panels should try to consider all the relevant factors. Additionally, EB-CPGs using GRADE are preferable for enhancing the EtR rigor.

\section{Background}

Clinical practice guidelines (CPGs) have been defined as "statements that include recommendations intended to optimize patient care that are informed by a systematic review of evidence and a risk-benefit assessment of alternative care options" [1]. CPGs have been playing an important role in guiding clinical decisions on prevention, diagnosis, treatment, and care [2]. Transparent and rigorous recommendations in the CPGs can not only help clinicians make appropriate decisions efficiently, but also ensure medical safety $[3,4]$. However, with the rapid development of evidence-based medicine, although the number of Chinese $\mathrm{CPGs}$ is growing rapidly, the rigor of formulating recommendations is lower than the international level [5-7]. 
The recommendations need to be systematically developed using the best available evidence, but highquality evidence of itself does not necessarily signify the high strength of recommendations $[4,8,9]$. The quality of evidence reflects the extent to which our confidence in an estimate of the effect is adequate to support a particular recommendation [8]. While the strength of a recommendation reflects the extent to which a guideline panel is confident that desirable effects of an intervention outweigh undesirable effects by considering all the relevant factors, including the effectiveness of the intervention, safety, feasibility, costs, values and preferences, equity and balance of benefits and harms that influence a recommendation in a rigorous, explicit, and transparent way, across the range of patients for whom the recommendation is intended [4, 8-12]. In the process of formulating recommendations, the guideline panel which consists of staff with relevant skills, perspectives, and knowledge will reach a consensus by discussing or voting $[3,4]$. At present, there have already been many supporting tools and models to standardize and facilitate the process of evidence to recommendations (EtR), such as the GRADE Evidence to Decision (EtD) framework, the GRADEPro Guideline Development Tool (GRADEPro GDT) (www.gradepro.org), which can help the guideline panel use evidence in a structured and transparent way to inform decisions in the context of clinical recommendations, coverage decisions, and health system or public health recommendations and decisions $[3,4,13]$. However, Chen et al [14] reported from examining 269 guidelines across China published from 1993 to 2010 that only one guideline used GRADE, while none of the CPGs referred to values and preferences of the patient, 2 guidelines $(<1 \%)$ were reviewed externally before publication, what's more, only 2 guidelines involved the participation of methodologists. Recently, many researchers have evaluated CPGs in China using the AGREE II instrument [6, 15-17]. But as far as we know, there are no studies focusing on exploring the methodological characteristics of EtR. The overall situation of EtR in Chinese CPGs development is unclear, especially in the recent decade.

This study aimed to describe the status and trends of the main methodological characteristics of EtR by analyzing all Chinese CPGs published during the period 2010-2020; compare the methodological characteristics of EtR of the Chinese CPGs in EB-CPGs with CB-CPGs, and GRADE with non-GRADE CPGs; determine the main methodological factors of the rigor of EtR in CPGs and then, make several suggestions for a rigorous EtR for CPGs.

\section{Methods}

\section{Search strategy}

The two main Chinese electronic databases were systematically searched, CNKI (China National Knowledge Infrastructure) and Wan Fang Database to identify CPGs. According to the title definition, we classified the CPGs into EB-CPGs and CB- CPGs. In this research, CB-CPGs (including consensus statements and expert opinions) refer to CPGs developed by guideline panels based on only low-quality or very low-quality evidence. The keywords for the searches included Chinese words for terms, such as "clinical practice guideline" or "practice guideline" or "clinical guideline" or "guideline" or "recommendation" or "consensus" or "consensus statement" or "expert consensus" or "expert consensus statement" in the titles. The search dates were from 1st January 2010 up to 31st December 2020. 


\section{Guideline Identification}

All CPGs were included if they met both of the following criteria: (1) The articles were considered as CPGs if they met the definition of a CPG as proposed by IOM [1, 18]; (2) The CPGs illustrated recognizable recommendations [12, 19-21]: ) The CPGs used Chinese words for terms or phrases such as "should", "strongly recommend", "suggest", "consider", "must", "could", "should not" to identify recommendations; ) The CPGs presented recommendations separately by using text boxes/summarizing tables, recommendations flow charts, or propositions of recommendations in executive summary; ) The CPGs presented recommendations in the form of italic or bold fonts.

If several versions of one CPG existed, only the version that included the greatest detail on the CPG development methodology was assessed. Where the same CPG was published in different journals simultaneously, only one was chosen. Where one CPG was published in several parts, we merged them into one complete CPG. The Chinese versions of foreign CPGs and adapted versions of CPGs from other countries were excluded. Articles were also excluded if the full text was unavailable.

\section{Screening and review}

Two reviewers (Yan SY, Ren XY) independently selected relevant guidelines as proposed by IOM, and screened eligible studies by title, abstract, and full text. Then, two reviewers (Zheng N, Ren XY) independently selected CPGs that specified recommendations. Discrepancies were resolved by consensus or discussion with a third researcher (Jin YH).

\section{Data Extraction}

To generate potential items for the data-extraction form for the methodological characteristics of EtR, we implemented a 3-aspect approach: (1) we extracted the main methodological characteristics associated with the process of EtR from the development manuals on CPGs, by accessing the websites of all major guideline databases, famous academic societies or institutions (Appendix A); (2) the GRADE [8](including the GRADE EtD framework [3, 4, 22, 23]), AGREE [24, 25](www.agreetrust.org), and the Guidelines 2.0 checklist $[9,26]$ were obtained to give the main methodological characteristics associated with the

process of EtR; (3) then face-to-face group meetings were held to discuss and confirm all potential items.

In addition to general characteristics (title, publication year), we also extracted 12 methodological characteristics in 4 domains of EtR from the included CPGs: (1) The basic characteristics: type of CPGs (EB-CPGs or CB-CPGs), grading system (GRADE or non-GRADE); (2) The relevant factors being considered: the effectiveness of the intervention, safety, feasibility, costs, values and preferences, equity, balance of benefits and harms. The CPGs considering relevant factors of EtR were defined as those CPGs that presented the information of cited references supporting the consideration of relevant factors in the text of the CPGs, such as original research or systematic reviews. In this study, greater than or equal to 3 relevant factors was considered as represented a rigorous process of EtR. (3) The information related to the process of consensus or voting: the consensus methods (Delphi Method, Nominal Group Technique, 
Consensus Development Conference, et al.) used [27], the holding of consensus meetings to discuss or vote on recommendations, stuff composition of consensus meetings, the participation of patients, the declaration of conflicts of interest, the production of Evidence Profiles (EP) or Summary of Findings (SoF) tables and the use of supporting tools (such as GRADE EtD framework or GRADEPro, et al); (4) Other characteristics: the designated level of evidence, and the conduct of external reviews.

Data were extracted using a double-extraction method from each eligible CPG and its corresponding appendices by two reviewers (Zheng N, Ren XY) who are familiar with Evidence-based medicine and CPG development methodology. Any disagreement was also resolved through discussion with a third author (Jin YH). Finally, two independent reviewers recorded the characteristics of included CPGs in an EXCEL file. Kappa statistics were calculated to evaluate inter-rater reliability between the two assessors using SPSS 23.0 software. Kappa value of $>0.75$ was considered as high inter-rater reliability.

\section{Statistical Analysis}

Categorical variables were described using frequencies and percentages. The data relating to methodological characteristics of EtR were summarized and stratified by the year of the CPGs development. The "color scale chart" was used to adjust the basic tones of the image to provide a visual reference for the reader. We used Mann Kendall Trend Test (M-K test), a non-parametric method, to identify monotonically increasing or decreasing trends of methodological characteristics over years, a positive $z$ value indicated a monotonic upward trend, and a negative one indicated a downward trend.

We classified included CPGs as CB-CPGs and EB-CPGs based on category and dichotomized CPGs based on whether or not GRADE was used. Chi-squared tests were conducted to assess differences in EBCPGs versus CB-CPGs and GRADE versus non-GRADE of CPGs from 2010-2020 in types of methodological characteristics.

Multi-factor Binary Logistic regression analysis was used to analyze factors related to the rigor of the process of EtR in the CPGs. The dependent variable was a rigorous process of EtR. The independent variables covered EB-CPGs or CB-CPGs, GRADE or non-GRADE CPGs, the designation of the level of evidence, the use of the consensus methods, the holding of consensus meetings to discuss or vote on recommendations, the participation of methodologists, the participation of patients, the declaration of conflicts of interest, the production of EP or SoF tables, the use of supporting tools and the conduct of external reviews. We use "Yes/No" to classify all the dependent and independent variables. For simplification, adjusted odds ratios (ORs) with corresponding $95 \%$ confidence interval $(95 \% \mathrm{Cl}$ ) were presented for each regression. We evaluated the screened risk factors by the partial chi-square statistic minus the predicted degrees of freedom to measure the importance of each screened risk factor. All statistical tests were 2-sided at a significance level of $P<0.05$. The $M-K$ test was conducted in $\mathrm{R}$, version 4.0.1 (R Foundation), and the rest of the analyses were carried out using the SPSS software, version 23.0.

\section{Results}




\section{Flow of included studies}

We identified a total of 29,186 articles of which 18,078 were considered potentially relevant; after screening using titles and abstracts, 2,873 CPGs were selected. In the end, a total of 753 CPGs (EBCPGs=386/1,127, CB-CPGs=367/1,527) were selected based on the selection criteria (Fig. 1). Unfortunately, over the 11 years, only $28.4 \%$ (753/2654) of the CPGs illustrated recognizable recommendations There was high agreement between the authors extracting the data (Kappa=0.89; 95\% $\mathrm{Cl} 0.73-0.84 ; P<0.001)$. The differences in data extraction were resolved by consensus or discussion with the third author.

\section{Number of CPGs that illustrated recognizable recommendations}

From 2010 to 2020, it was evident that the production of CPGs specifying recognizable recommendations was increasing annually (Fig. 2A). The number of CB-CPGs illustrating recognizable recommendations published in the last two years was far higher than that of EB-CPGs.

\section{Trends of the methodological characteristics of EtR}

According to the result of $\mathrm{M}-\mathrm{K}$ test, there was an increasing tendency in these 5 characteristics $(P<0.05)$ in all the CPGs, including the consideration of relevant factors, the holding of consensus meetings to discuss or vote on recommendations, the participation of methodologists, the participation of patients, and the declaration of conflicts of interest (Fig. 2B). During the last 11 years, 43.7\% (329/753) of CPGs considered relevant factors. It's worth noting that just $24.4 \%$ (184/753) of CPGs used GRADE to develop recommendations.

As Fig. $2 \mathrm{C}$ shows, the EB-CPGs have improved in the 6 characteristics over the time-span by M-K test $(P<0.05)$, which include the consideration of relevant factors, GRADE or non-GRADE CPGs, the participation of methodologists, the participation of patients, the declaration of conflicts of interest, and the conduct of external reviews. As reported, $52.6 \%$ (203/386) of EB-CPGs considered relevant factors with an increasing trend $(P<0.05)$. Although there were only $29.0 \%(112 / 386)$ of EB-CPGs using GRADE to develop recommendations, the proportion of EB-CPGs using GRADE has still been increasing year on year $(P<0.05)$.

As presented in Fig. 2D, 5 characteristics showed an increasing tendency over the 11 years based on the $\mathrm{M}-\mathrm{K}$ test in $\mathrm{CB}-\mathrm{CPGs}(P<0.05)$. The 5 characteristics referred to the consideration of relevant factors, the use of consensus methods, the holding of consensus meetings to discuss or vote on recommendations, the declaration of conflicts of interest, and the conduct of external reviews. Although there were only $34.3 \%(126 / 367)$ of CB-CPGs considering the relevant factors, which was less than that in EB-CPGs, a significant improvement was observed in both EB-CPGs and CB-CPGs in this area over the time span $(P<0.05)$. Unfortunately, the proportion of CB-CPGs using GRADE to develop recommendations was only $19.6 \%$ (72/367), which was also less than EB-CPGs, and there was no significant upward trend in this area in CB-CPGs $(P>0.05)$. 


\section{The information about the relevant factors being considered}

The factors considered most were the effectiveness of interventions $(398,52.9 \%)$, safety $(368,48.9 \%)$, and balance of benefits and harms $(193,25.6 \%)$. However, across all the 753 CPGs, there were only 95 CPGs which considered costs, the proportion was even lower for consideration of values and preferences $(57,7.6 \%)$ and feasibility $(47,6.2 \%)$ no $C P G$ considered fairness.

\section{Methodological characteristics of EtR in EB-CPGs versus CB-CPGs}

In total, there were statistically significant differences in 10 characteristics demonstrated by Chi-squared test $(P<0.05)$ (Fig. 3A), including the consideration of relevant factors, GRADE or non-GRADE CPGs, the designation of the level of evidence, the use of consensus methods, the holding of consensus meetings to discuss or vote on recommendations, the participation of methodologists, the participation of patients, the declaration of conflicts of interest, the production of EP or SoF tables and the conduct of external reviews.

From 2010 to 2020, there were 184 (24.4\%) CPGs that used GRADE to develop recommendations, which was $29.0 \%$ (112/386) EB-CPGs and 19.6\% (72/367) CB-CPGs respectively. As presented in Fig. 3A, there were no CB-CPGs producing EP or SoF tables, or CB-CPGs using the supporting tools. Moreover, the proportion of CB-CPGs involving patient participation was $0.3 \%(1 / 367)$, and only $2.2 \%(8 / 367)$ of CBCPGs were developed with the participation of methodologists. Only $0.5 \%(2 / 367)$ of CB-CPGs conducted external reviews. What's more, the CB-CPGs using the consensus methods accounted for $7.6 \%(28 / 367)$ of the total number of publications, and 9.5\% (35/367) of CB-CPGs held the consensus meetings to discuss or vote on recommendations. Fortunately, in terms of all the methodological characteristics of EtR, EB-CPGs did better than CB-CPGs. However, in EB-CPGs, the proportion using supporting tools (1.3\%, $5 / 386)$, those producing EP or SoF tables $(3.1 \%, 12 / 386)$, those holding consensus meetings to discuss or vote on recommendations $(5.7 \%, 22 / 386)$ and those involving the participation of patients $(8.8 \%$, $34 / 386)$ was still lower than $10.0 \%$.

\section{Methodological characteristics of EtR using GRADE versus non-GRADE}

According to the statistics, a total of 184 (24.4\%) CPGs used GRADE, and 75.6\% (569/753) did not. As Fig. 3B shows, compared with CPGs not using GRADE, we observed higher methodological quality in CPGs using GRADE across the 10 methodological characteristics $(P<0.05)$, which included the consideration of relevant factors, EB-CPGs or CB-CPGs, the designation of the level of evidence, the use of consensus methods, the holding of consensus meetings to discuss or vote on recommendations, the participation of methodologists, the declaration of conflicts of interest, the use of supporting tools, the production of EP or SoF tables, and the conduction of external reviews.

Unexpectedly, none of the non-GRADE CPGs employed supporting tools, EP or SoF tables. Furthermore, only $3.9 \%(22 / 569)$ of the CPGs were developed with the participation of methodologists, and only $4.0 \%$ 
$(23 / 569)$ were developed with the participation of patients. Compared with the non-GRADE CPGs, the CPGs using GRADE did a better job in area of the participation of methodologists $(26.1 \%, 48 / 184)$. It is satisfying that, $92.4 \%(170 / 184)$ of the CPGs using GRADE designated the level of evidence, and $64.7 \%$ $(119 / 184)$ of the CPGs using GRADE were developed more explicitly, and transparently by presenting evidence of considering the factors. Last but not least, it's worth noting that $60.9 \%(112 / 184)$ of CPGs using GRADE were EB-CPGs.

\section{Factors associated with the rigor of EtR}

The independent factors associated with the rigor of EtR in the Multi-factor Binary Logistic regression analysis are detailed in Fig. 3C. More CPGs using GRADE may have considered the relevant factors than those not (OR 2.03, 95\% Cl 1.37-3.00, $P<0.001$ ). Compared with CB-CPGs, EB-CPGs were more likely to consider the relevant factors (OR $1.48,95 \% \mathrm{Cl} 1.06-2.07, P=0.022)$. Similar findings were seen in terms of the designation of the level of evidence (OR 1.64, 95\% $\mathrm{Cl} 1.15-2.34, P=0.006)$. By the partial chi-square statistic minus the predicted degrees of freedom, we find that CPGs using GRADE was the most important factor associated with the rigor of EtR.

\section{Discussion}

This survey shows that the process and EtR in Chinese CPGs over the last 11 years is not satisfactory, especially in terms of the consideration of relevant factors. A number of recommendations were developed without sufficient consideration of feasibility, costs, values and preferences or balance of benefits and harms, which is similar to findings by Chen YL, et al [14]. What's more, according to the result of the Chi-square test and the Multi-factor Binary Logistic regression analysis, the CPGs using GRADE outperformed those that did not, and the EB-CPGs were superior to the CB-CPGs across almost all methodological characteristics of EtR involved.

The authoritative academic societies or institutions had made it clear that to formulate a recommendation, it's important to take the relevant factors into consideration, such as values and preferences related to the outcomes of an intervention or exposure, balance of benefits and harms and acceptability, which have different degrees of influence on the process of EtR, and sometimes even play a decisive role $[8,10,12,26]$. These considerations mean that high certainty evidence does not necessarily imply strong recommendations, and strong recommendations can result from low or even very low certainty of evidence by taking all the relevant factors together [28]. In addition, to make a recommendation, a panel must consider the implication and importance of each of the relevant factors to make sure that when there is uncertainty or disagreement, it can help to explicitly consider this for each criterion [4]. While the neglect of relevant factors of EtR may affect the credibility and acceptability of recommendations, this disadvantages the promotion and implementation of the CPGs [29, 30]. The structure and standardization of Chinese CPGs are still gradually developing. A large proportion of CPGs in the early stage, focused on health effects ignoring important criteria for decision-making including feasibility, cost, acceptability, and equity of the interventions $[6,14]$. What's more, the 
methodologist also plays a critical role in EtR by helping the guideline panel to formulate recommendations informed by the evidence in a transparent and explicit manner [10, 12]. Unfortunately, it is reported that the CPGs rarely involved patients' participation in the process of EtR, which has led to the neglect of patient's perspectives [14,31]. This study confirmed these conclusions. Moreover, the absence of methodologists in the process of EtR may also lead to the neglect of evidence regarding patients' value and preferences, costs, and feasibility, which will reduce the rigor of the EtR process. Therefore, the guidelines working group should specify and accurately define the relevant factors that need to be taken into account when formulating recommendations, and assess the criteria of relevant factors being considered quantitatively as much as possible. What's more, guideline developers should also use supporting tools or frameworks to ensure that recommendations are scientifically and transparently formulated.

It is acknowledged that the use of GRADE to develop recommendations is an important safeguard of the structural factors, transparency, and rigor of CPGs, and this has been confirmed by our study [32, 33]. Because of the loose methodological requirements for the formulation of CB-CPGs, the development process of CB-CPGs has usually been much simpler and faster, possibly leading to significantly worse methodological quality than that of EB-CPGs [6]. This study has confirmed that the EB-CPGs are superior to the $\mathrm{CB}-\mathrm{CPGs}$ in the vast majority of methodological characteristics related to methodological quality of EtR. Therefore, the EB-CPGs using GRADE based on strict methodology rather than CB-CPGs should be selected as far as possible when considering the actual situation.

We have also made the following suggestions: (1) recommendations in the CPGs should be based on a systematic review of the scientific literature guided by specific key questions about the intervention, exposure or approach under consideration, which should underpin all recommendations on the effectiveness of the intervention, safety, feasibility, costs, values and preferences, equity, balance of benefits and harms; (2) it is necessary to include methodologists into the guideline panels to regulate the process of EtR; (3) we should attach importance to the rigor of formulating recommendations to promote the implementation and promotion of the CPGs.

\section{Strengths and limitations}

As far as we are aware, this is the first systematic review on the status of EtR in Chinese CPGs, which is based on a systematic search of all Chinese CPGs for the last 11 years. The data-extraction form based has been based on the authoritative guidance documents including the CPGs development manuals, GRADE and AGREE websites or papers, and the Guidelines 2.0 checklist. The data-extraction form has covered most of the important characteristics that may be relevant in the process of EtR. We also found several most important factors related to the rigor of EtR in CPGs. Overall, our work provides the basis for these important next steps in improving the methodological quality of EtR in Chinese CPGs.

This systematic review has certain limitations. Evaluating the methodological quality of CPGs, we simply analyzed the data reported in the literature. Some items may not have been fully reported due to reporting differences or space restrictions of publishers. Unfortunately, our study did not contact authors to obtain 
further access and analysis. Consequently, the conclusion of this research may underestimate the methodological quality of EtR in Chinese CPGs.

\section{Conclusion}

The CPGs have played a vital role in clinical medicine, and have already become one of the most important tools for potentially improving clinical decision-making, patients' outcomes and reducing medical costs. It is very important to develop recommendations rigorously. The rigor of EtR in CPGs will not only improve the overall quality of the guidelines but also facilitate their dissemination and implementation. However, the status of relevant factors being considered in the process of EtR in Chinese CPGs across the last 11 years is not satisfactory. It is critical to consider not only evidence of the effects of an intervention on health outcomes, but also all relevant factors by rigorous methodology for guideline panels to reach the consensus. Further exploration and development for the impact of relevant factors on the feasibility of the implementation of CPGs are still needed.

\section{Abbreviations}

EtR: The process of moving from evidence to recommendations; CPGs: Clinical practice guidelines; EtD: Evidence to Decision; GRADEPro GDT: The GRADEPro Guideline Development Tool; CNKI: China National Knowledge Infrastructure; EP: Evidence Profiles; SoF: Summary of Findings; M-K test: Mann Kendall Trend Test.

\section{Declarations}

\section{Acknowledgements}

The authors wish to thank the members who participated in the study.

\section{Authors' contributions}

(I) Conception and design: N Zheng, YJ Cao, YH Jin; (II) Administrative support: XM Chen, YJ Cao, YH Jin; (III) Provision of study materials or patients: N Zheng, XY Ren, R Tan, LX Su; (IV) Collection and assembly of data: N Zhen, XY Ren, YH Jin; (V) Data analysis and interpretation: N Zheng, XY Guan, Q Huang; (VI) Manuscript writing: N Zheng, YH Jin; (VII) Final approval of manuscript: All authors.

\section{Funding}

No funding was provided for this paper

\section{Availability of data and materials}

The datasets analyzed during the current study are available from the corresponding author on reasonable request. 


\section{Ethics approval and consent to participate}

Not applicable. This study did not involve human participants. We analyzed publicly available information from scholarly journals and public web sites.

\section{Consent for publication}

Not applicable.

\section{Competing interests}

The authors declare no competing financial interests.

\section{References}

1. Institute of Medicine (US) Committee on Standards for Developing Trustworthy Clinical Practice Guidelines, Graham R, Mancher M, Miller Wolman D, Greenfield S, Steinberg E, eds. Clinical Practice Guidelines We Can Trust. Washington (DC): National Academies Press (US); 2011.

2. Wang YY, Huang Q, Shen Q, Zi H, Li BH, Li MZ et al: Quality of and Recommendations for Relevant Clinical Practice Guidelines for COVID-19 Management: A Systematic Review and Critical Appraisal. Front Med 2021, 8:630765. https://doi.org/10.3389/fmed.2021.630765.

3. Alonso-Coello P, Schünemann HJ, Moberg J, Brignardello-Petersen R, AkI EA, Davoli M et al: GRADE Evidence to Decision (EtD) frameworks: a systematic and transparent approach to making well informed healthcare choices. 1: Introduction. BMJ 2016, 353:i2016. https://doi.org/10.1136/bmj.i2016.

4. Alonso-Coello P, Oxman AD, Moberg J, Brignardello-Petersen R, Akl EA, Davoli M et al: GRADE Evidence to Decision (EtD) frameworks: a systematic and transparent approach to making well informed healthcare choices. 2: Clinical practice guidelines. BMJ 2016, 353:i2089. https://doi.org/10.1136/bmj.i2089.

5. Zheng ZH, Cui SQ, Lu XQ, Zakus D, Liang WN, Huang F et al: Analysis of the status of Chinese clinical practice guidelines development. BMC Health Serv Res 2012, 12:218. https://doi.org/10.1186/1472-6963-12-218.

6. Chen Y, Wang C, Shang H, Yang K, Norris SL: Clinical practice guidelines in China. Bmj 2018, 360:j5158. https://doi.org/10.1136/bmj.j5158.

7. Zhang S, Wu L, Wang Y, Zhou Q, Luo X, Mathew JL, Wang Q, Song Y, Chen Y: Methodological and reporting quality of pediatric clinical practice guidelines: a systematic review. Ann Transl Med 2021, 9:1258. https://doi.org/10.21037/atm-21-2686.

8. Sch Unemann Holger BJGG: GRADE Handbook. In.; 2013.

9. Schünemann F, Meerpohl JJ, Schwingshackl L, Kopp IB, Schünemann HJ: [Guidelines 2.0:

systematic development of a comprehensive checklist for a successful guideline enterprise]. Z Evid 
Fortbild Qual Gesundhwes 2021, 163:76-84. https://doi.org/10.1016/j.zefq.2021.01.009.

10. Organization WH: WHO handbook for guideline development, 2nd ed. 2014

11. Atkins D, Best D, Briss PA, Eccles M, Falck-Ytter Y, Flottorp S et al: Grading quality of evidence and strength of recommendations. BMJ 2004, 328:1490. https://doi.org/10.1136/bmj.328.7454.1490.

12. Excellence TNIfHaC: Developing NICE guidelines: the manual. 2014

13. Skoetz N, Goldkuhle M, van Dalen EC, Akl EA, Trivella M, Mustafa RA et al: GRADE guidelines 27: how to calculate absolute effects for time-to-event outcomes in summary of findings tables and Evidence Profiles. J Clin Epidemiol 2020, 118:124-131. https://doi.org/10.1016/j.jclinepi.2019.10.015.

14. Chen YL, Yao L, Xiao XJ, Wang Q, Wang ZH, Liang FX et al: Quality assessment of clinical guidelines in China: 1993 - 2010. Chin Med J (Engl) 2012, 125:3660-3664

15. Lu S, Luo X, Ni X, Li H, Meng M, Cai Y et al: Reporting quality evaluation of the stroke clinical practice guidelines: a systematic review. Syst Rev 2021, 10:262. https://doi.org/10.1186/s13643-021-018053.

16. Yao S, Wei D, Chen YL, Wang Q, Wang XQ, Zeng Z, Li H: Quality assessment of clinical practice guidelines for integrative medicine in China: A systematic review. Chin J Integr Med 2017, 23:381385. https://doi.org/10.1007/s11655-016-2739-z.

17. Li CY, Chen YL, Hu JY, Li M, Zhang XY, Sun Y et al: Status Quo and Analysis of the Cardiovascular Clinical Practice Guidelines/Expert Consensuses of Chinese and Integrative Medicine: A Systematic Review. Chin J Integr Med 2021, 27:54-61. https://doi.org/10.1007/s11655-020-2725-3.

18. Medicine lo: Clinical Practice Guidelines: Directions for a New Program. Washington, DC: The National Academies Press; 1990.

19. Free SA, Delp SL: Trochanteric transfer in total hip replacement: effects on the moment arms and force-generating capacities of the hip abductors. J Orthop Res 1996, 14:245-250. https://doi.org/10.1002/jor.1100140212.

20. NSGC: Evidence-Based Clinical Practice Guideline Development Manual. 2016

21. IDSA: Handbook on Clinical Practice Guideline Development. . 2013

22. DECIDE: Evidence to Decision (EtD) framework.

23. Moberg J A-CP, Oxman AD. : GRADE Evidence toDecision (EtD) Frameworks Guidance. Version 1.1[updated May 2015]. The GRADE Working Group 2015

24. Brouwers MC, Kho ME, Browman GP, Burgers JS, Cluzeau F, Feder G et al: AGREE II: advancing guideline development, reporting and evaluation in health care. CMAJ 2010, 182:E839-842. https://doi.org/10.1503/cmaj.090449.

25. Burls A: AGREE II-improving the quality of clinical care. Lancet 2010, 376:1128-1129. https://doi.org/10.1016/s0140-6736(10)61034-3.

26. Schünemann HJ, Wiercioch W, Etxeandia I, Falavigna M, Santesso N, Mustafa R et al: Guidelines 2.0: systematic development of a comprehensive checklist for a successful guideline enterprise. CMAJ 2014, 186:E123-142. https://doi.org/10.1503/cmaj.131237. 
27. Eccles M, Clapp Z, Grimshaw J, Adams PC, Higgins B, Purves I, Russell I: North of England evidence based guidelines development project: methods of guideline development. BMJ 1996, 312:760-762. https://doi.org/10.1136/bmj.312.7033.760.

28. Yao L, Brignardello-Petersen R, Guyatt GH: Developing trustworthy guidelines using GRADE. Can J Ophthalmol 2020, 55:349-351. https://doi.org/10.1016/j.jcjo.2020.09.001.

29. Flodgren G, Hall AM, Goulding L, Eccles MP, Grimshaw JM, Leng GC, Shepperd S: Tools developed and disseminated by guideline producers to promote the uptake of their guidelines. Cochrane Database Syst Rev 2016:Cd010669. https://doi.org/10.1002/14651858.CD010669.pub2.

30. Flottorp SA, Oxman AD, Krause J, Musila NR, Wensing M, Godycki-Cwirko M, Baker R, Eccles MP: A checklist for identifying determinants of practice: a systematic review and synthesis of frameworks and taxonomies of factors that prevent or enable improvements in healthcare professional practice. Implement Sci 2013, 8:35. https://doi.org/10.1186/1748-5908-8-35.

31. WANG Zijun, SHI Qianling, LIU Yunlan, REN Mengjuan, ZHAO Siya, MA Yanfang et al: Investigation and Evaluation of Chinese Clinical Practice Guidelines Published in Medical Journals in 2019: Status of the Authorship and Guideline Development Group. Medical Journal of Peking Union Medical College Hospital 2021, 12:552-559

32. Wang X, Zhou Q, Chen Y, Yang N, Pottie K, Xiao Y et al: Using RIGHT (Reporting Items for Practice Guidelines in Healthcare) to evaluate the reporting quality of WHO guidelines. Health Res Policy Syst 2020, 18:75. https://doi.org/10.1186/s12961-020-00578-w.

33. Barker TH, Dias M, Stern C, Porritt K, Wiechula R, Aromataris E, Brennan S, Schünemann HJ, Munn Z: Guidelines rarely used GRADE and applied methods inconsistently: A methodological study of Australian guidelines. J Clin Epidemiol 2021, 130:125-134.

https://doi.org/10.1016/j.jclinepi.2020.10.017.

\section{Figures}




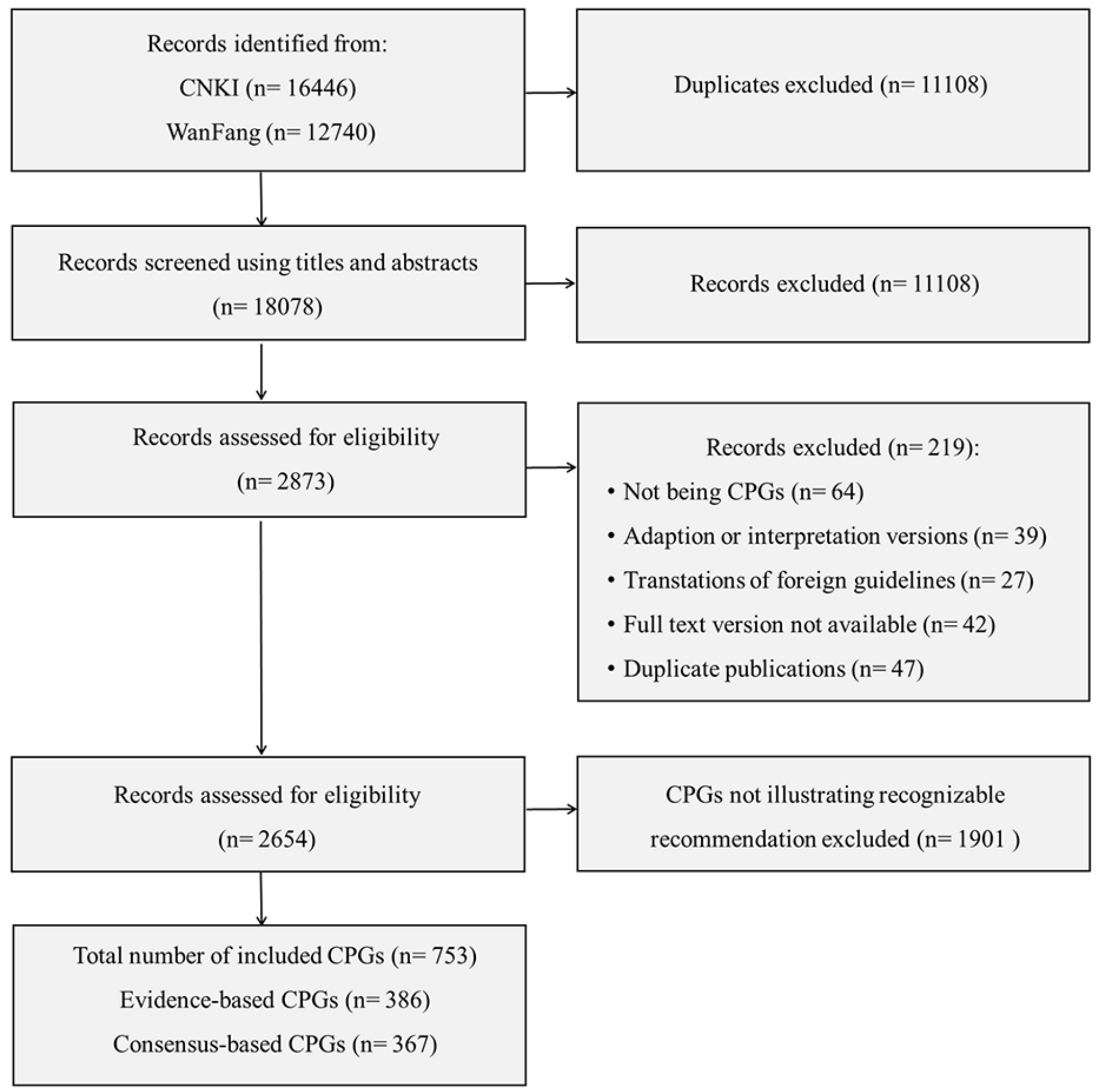

\section{Figure 1}

The flowchart for CPGs selection. 
A The publication trend of the CPGs illustrating recognizable recommendations during 2010-2020 $=\mathrm{CB}$-CPGs $=\mathrm{EB}$-CPGs $\bullet$ CPGs

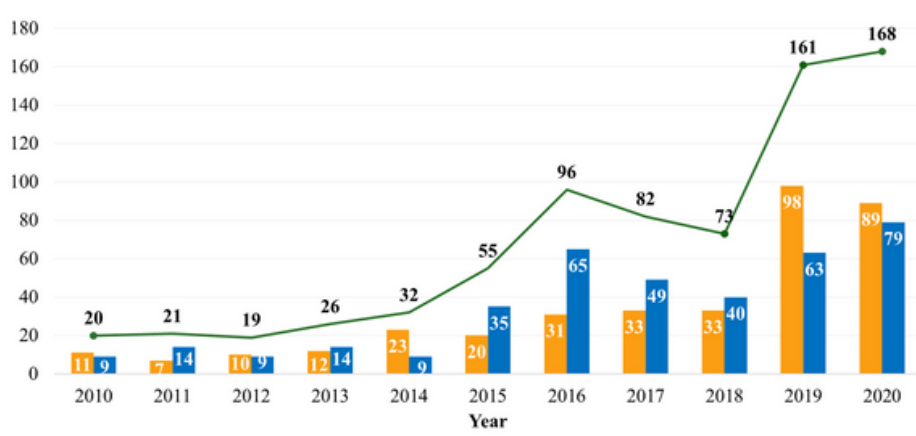

C Methodological characteristics of EtR in Chinese EB-CPGs over 11 years \begin{tabular}{l|l|l|l|l|l|l|l|l|l|lll}
2010 & 2011 & 2012 & 2013 & 2014 & 2015 & 2016 & 2017 & 2018 & 2019 & 2020 & $\mathrm{z}$ & $P$
\end{tabular}

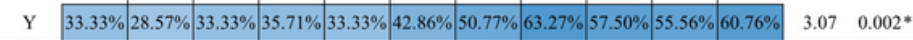
\begin{tabular}{l|l|l|l|l|l|l|l|l|l|l|ll|l}
\hline A & $0.00 \%$ & $14.29 \%$ & $0.00 \%$ & $0.00 \%$ & $11.11 \%$ & $14.29 \%$ & $26.15 \%$ & $16.33 \%$ & $47.50 \%$ & $38.10 \%$ & $45.57 \%$ & 3.00 & $0.003 *$ \\
\hline
\end{tabular}

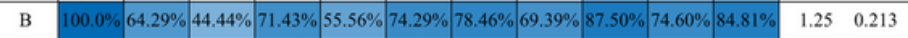

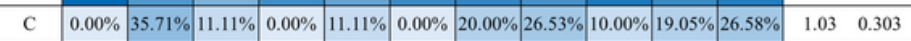
\begin{tabular}{l|l|l|l|l|l|l|l|l|l|l|l|ll}
\hline D & $0.00 \%$ & $7.14 \%$ & $0.00 \%$ & $0.00 \%$ & $11.11 \%$ & $5.71 \%$ & $3.08 \%$ & $8.16 \%$ & $2.50 \%$ & $4.76 \%$ & $10.13 \%$ & 1.26 & 0.206 \\
\hline
\end{tabular} \begin{tabular}{c|c|c|c|c|c|c|c|c|c|c|c|cc}
\hline E & $0.00 \%$ & $7.14 \%$ & $0.00 \%$ & $0.00 \%$ & $11.11 \%$ & $0.00 \%$ & $9.23 \%$ & $14.29 \%$ & $17.50 \%$ & $20.63 \%$ & $34.18 \%$ & 3.04 & $0.002 *$ \\
\hline
\end{tabular} \begin{tabular}{c|c|c|c|c|c|c|c|c|c|c|c|cc|}
\hline F & $0.00 \%$ & $0.00 \%$ & $0.00 \%$ & $0.00 \%$ & $11.11 \%$ & $0.00 \%$ & $13.85 \%$ & $22.45 \%$ & $2.50 \%$ & $6.35 \%$ & $10.13 \%$ & 1.97 & $0.049 *$ \\
\hline
\end{tabular} \begin{tabular}{c|c|c|c|c|c|c|c|c|c|c|c|c|c|c|}
\hline G & $0.00 \%$ & $28.57 \%$ & $11.11 \%$ & $0.00 \%$ & $11.11 \%$ & $2.86 \%$ & $38.46 \%$ & $44.90 \%$ & $30.00 \%$ & $63.49 \%$ & $54.43 \%$ & 2.51 & $0.012 *$ \\
\hline
\end{tabular} \begin{tabular}{c|c|c|c|c|c|c|c|c|c|c|c|cc}
\hline $\mathrm{H}$ & $0.00 \%$ & $7.14 \%$ & $0.00 \%$ & $0.00 \%$ & $0.00 \%$ & $0.00 \%$ & $3.08 \%$ & $0.00 \%$ & $5.00 \%$ & $4.76 \%$ & $5.06 \%$ & 1.39 & 0.165 \\
\hline
\end{tabular} \begin{tabular}{c|c|c|c|c|c|c|c|c|c|c|c|cc}
\hline I & $0.00 \%$ & $0.00 \%$ & $0.00 \%$ & $0.00 \%$ & $0.00 \%$ & $0.00 \%$ & $3.08 \%$ & $2.04 \%$ & $2.50 \%$ & $0.00 \%$ & $1.27 \%$ & 1.65 & 0.100 \\
\hline
\end{tabular}

\begin{tabular}{l|l|l|l|l|l|l|l|l|l|l|l|l|l|}
$\mathrm{J}$ & $0.00 \%$ & $14.29 \%$ & $0.00 \%$ & $0.00 \%$ & $0.00 \%$ & $0.00 \%$ & $13.85 \%$ & $22.45 \%$ & $5.00 \%$ & $17.46 \%$ & $17.72 \%$ & 2.06 & $0.039 *$
\end{tabular} \begin{tabular}{l|l|l|l|l|l|l|l|l|l|l}
$\mathrm{N}=9$ & $\mathrm{~N}=14$ & $\mathrm{~N}=9$ & $\mathrm{~N}=14$ & $\mathrm{~N}=9$ & $\mathrm{~N}=35$ & $\mathrm{~N}=65$ & $\mathrm{~N}=49$ & $\mathrm{~N}=40$ & $\mathrm{~N}=63$ & $\mathrm{~N}=79$
\end{tabular}

Y Considered relevant factors

A GRADE or non-GRADE CPGs

B The level of evidence designated

C The use of the consensus methods

D The holding of consensus meetings to discuss or vote on recommendations

E The participation of methodologists
B Methodological characteristics of EtR in Chinese CPGs over 11 years \begin{tabular}{l|l|l|l|l|l|l|l|l|l|lll}
2010 & 2011 & 2012 & 2013 & 2014 & 2015 & 2016 & 2017 & 2018 & 2019 & 2020 & $\mathrm{z}$ & $P$
\end{tabular}

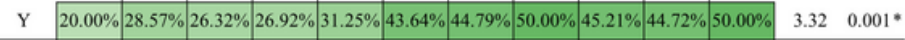

\begin{tabular}{|c|c|c|c|c|c|c|c|c|c|c|c|c|c|}
\hline $\mathrm{A}$ & & & & & & & & & & & & & \\
\hline B & & & & & & & & & & & & & \\
\hline C & $0.00 \%$ & $23.81 \%$ & $5.26 \%$ & $0.00 \%$ & $6.25 \%$ & $0.00 \%$ & $13.54 \%$ & $20.73 \%$ & $13.70 \%$ & $12.42 \%$ & $17.86 \%$ & 1.26 & 0.206 \\
\hline D & $.00 \%$ & $76 \%$ & $0.00 \%$ & $3.85 \%$ & $3.13 \%$ & $3.64 \%$ & $5.21 \%$ & $7.32 \%$ & $9.59 \%$ & $7.45 \%$ & & 2.99 & $0.003^{*}$ \\
\hline E & $0 \%$ & $6 \%$ & $00 \%$ & $0.00 \%$ & $13 \%$ & $.00 \%$ & $25 \%$ & $8.54 \%$ & $13.70 \%$ & $8.07 \%$ & 19.0 & 2.72 & $0.007^{*}$ \\
\hline $\mathrm{F}$ & $0.00 \%$ & $0.00 \%$ & $0.00 \%$ & $0.00 \%$ & $3.13 \%$ & $0.00 \%$ & $9.38 \%$ & $13.41 \%$ & $2.74 \%$ & $2.48 \%$ & $4.76 \%$ & 2.06 & $0.039^{*}$ \\
\hline G & $0.00 \%$ & $19.05 \%$ & $5.26 \%$ & $0.00 \%$ & $3.13 \%$ & $1.82 \%$ & $30.21 \%$ & $30.49 \%$ & $19.18 \%$ & $15404^{\circ}$ & $31.55 \%$ & 2.28 & $0.023^{*}$ \\
\hline H & $0.00 \%$ & $4.76 \%$ & $0.00 \%$ & $0.00 \%$ & $0.00 \%$ & $0.00 \%$ & $2.08 \%$ & $0.00 \%$ & $2.74 \%$ & $1.86 \%$ & $2.38 \%$ & 1.04 & 0.298 \\
\hline I & $0.00 \%$ & $0.00 \%$ & $0.00 \%$ & $0.00 \%$ & $0.00 \%$ & $0.00 \%$ & $2.08 \%$ & $1.22 \%$ & $1.37 \%$ & $0.00 \%$ & $0.60 \%$ & 1.66 & 0.096 \\
\hline \multirow[t]{2}{*}{ J } & $0.00 \%$ & $9.52 \%$ & $0.00 \%$ & $0.00 \%$ & $0.00 \%$ & $0.00 \%$ & $9.38 \%$ & $13.41 \%$ & $2.74 \%$ & $7.45 \%$ & $8.93 \%$ & \multirow[t]{2}{*}{1.40} & \multirow[t]{2}{*}{0.161} \\
\hline & $\mathrm{N}=20$ & $\mathrm{~N}=21$ & $\mathrm{~N}=19$ & $N=26$ & $\mathrm{~N}=32$ & $\mathrm{~N}=55$ & $\mathrm{~N}=96$ & $\mathrm{~N}=82$ & $\mathrm{~N}=73$ & $\mathrm{~N}=161$ & $\mathrm{~N}=1$ & & \\
\hline
\end{tabular}

D Methodological characteristics of EtR in Chinese CB-CPGs over 11 years \begin{tabular}{l|l|l|l|l|l|l|l|l|l|lll}
2010 & 2011 & 2012 & 2013 & 2014 & 2015 & 2016 & 2017 & 2018 & 2019 & 2020 & $\mathrm{z}$ & $P$
\end{tabular}

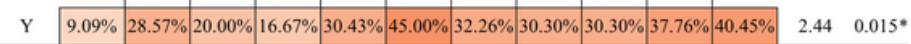
\begin{tabular}{l|l|l|l|l|l|l|l|l|l|l|l|l|l}
\hline $\mathrm{A}$ & $9.09 \%$ & $0.00 \%$ & $0.00 \%$ & $0.00 \%$ & $34.78 \%$ & $60.00 \%$ & $22.58 \%$ & $18.18 \%$ & $15.15 \%$ & $18.37 \%$ & $16.85 \%$ & 0.79 & 0.430 \\
\hline
\end{tabular} \begin{tabular}{l|l|l|l|l|l|l|l|l|l|l|l|l|l|l|l|l}
\hline B & $54.55 \%$ & $28.57 \%$ & $40.00 \%$ & $58.33 \%$ & $78.26 \%$ & $80.00 \%$ & $41.94 \%$ & $36.36 \%$ & $39.39 \%$ & $38.78 \%$ & $47.19 \%$ & -0.08 & 0.938 \\
\hline
\end{tabular} \begin{tabular}{c|c|c|c|c|c|c|c|c|c|c|c|cc|cc}
\hline $\mathrm{C}$ & $0.00 \%$ & $0.00 \%$ & $0.00 \%$ & $0.00 \%$ & $4.35 \%$ & $0.00 \%$ & $0.00 \%$ & $12.12 \%$ & $18.18 \%$ & $8.16 \%$ & $10.11 \%$ & 2.31 & $0.021^{*}$ \\
\hline
\end{tabular} \begin{tabular}{l|l|l|l|l|l|l|l|l|l|l|l|ll}
\hline $\mathrm{D}$ & $0.00 \%$ & $0.00 \%$ & $0.00 \%$ & $8.33 \%$ & $0.00 \%$ & $0.00 \%$ & $9.68 \%$ & $6.06 \%$ & $18.18 \%$ & $9.18 \%$ & $15.73 \%$ & 2.46 & $0.014^{*}$ \\
\hline
\end{tabular} \begin{tabular}{c|c|c|c|c|c|c|c|c|c|c|c|cc}
\hline E & $0.00 \%$ & $0.00 \%$ & $0.00 \%$ & $0.00 \%$ & $0.00 \%$ & $0.00 \%$ & $0.00 \%$ & $0.00 \%$ & $9.09 \%$ & $0.00 \%$ & $5.62 \%$ & 1.64 & 0.101 \\
\hline
\end{tabular} \begin{tabular}{c|c|c|c|c|c|c|c|c|c|c|c|cc}
\hline $\mathrm{F}$ & $0.00 \%$ & $0.00 \%$ & $0.00 \%$ & $0.00 \%$ & $0.00 \%$ & $0.00 \%$ & $0.00 \%$ & $0.00 \%$ & $3.03 \%$ & $0.00 \%$ & $0.00 \%$ & 0.79 & 0.429 \\
\hline
\end{tabular} \begin{tabular}{c|c|c|c|c|c|c|c|c|c|c|c|cc|c}
\hline G & $0.00 \%$ & $0.00 \%$ & $0.00 \%$ & $0.00 \%$ & $0.00 \%$ & $0.00 \%$ & $12.90 \%$ & $9.09 \%$ & $6.06 \%$ & $47.96 \%$ & $11.24 \%$ & 2.48 & $0.013 *$ \\
\hline
\end{tabular} \begin{tabular}{l|l|l|l|l|l|l|l|l|l|l|l|l}
\hline $\mathrm{H}$ & $0.00 \%$ & $0.00 \%$ & $0.00 \%$ & $0.00 \%$ & $0.00 \%$ & $0.00 \%$ & $0.00 \%$ & $0.00 \%$ & $0.00 \%$ & $0.00 \%$ & $0.00 \%$ & - \\
\hline
\end{tabular} \begin{tabular}{|l|l|l|l|l|l|l|l|l|l|l|l|l|l}
\hline $\mathrm{I}$ & $0.00 \%$ & $0.00 \%$ & $0.00 \%$ & $0.00 \%$ & $0.00 \%$ & $0.00 \%$ & $0.00 \%$ & $0.00 \%$ & $0.00 \%$ & $0.00 \%$ & $0.00 \%$ & - & - \\
\hline
\end{tabular} \begin{tabular}{l|l|l|l|l|l|l|l|l|l|l|l|rr}
$\mathrm{J}$ & $0.00 \%$ & $0.00 \%$ & $0.00 \%$ & $0.00 \%$ & $0.00 \%$ & $0.00 \%$ & $0.00 \%$ & $0.00 \%$ & $0.00 \%$ & $1.02 \%$ & $1.12 \%$ & 2.00 & $0.045 *$ \\
\cline { 2 - 9 }
\end{tabular} \begin{tabular}{l|l|l|l|l|l|l|l|l|l|l}
$\mathrm{N}=11$ & $\mathrm{~N}=7$ & $\mathrm{~N}=10$ & $\mathrm{~N}=12$ & $\mathrm{~N}=23$ & $\mathrm{~N}=20$ & $\mathrm{~N}=31$ & $\mathrm{~N}=33$ & $\mathrm{~N}=33$ & $\mathrm{~N}=98$ & $\mathrm{~N}=89$
\end{tabular}

F The participation of patient

$\mathrm{G}$ The declaration of conflicts of interest

$\mathrm{H}$ The production of EP or SoF tables

I The use of supporting tools

J The conduct of external reviews

${ }^{*} \mathrm{P}<0.05$

\section{Figure 2}

Methodological characteristics of EtR in Chinese CB-CPGs during 2010-2020. (A) The publication trend of the CPGs illustrating recognizable recommendations during 2010-2020. (B) Methodological characteristics of EtR in Chinese CPGs over 11 years. (C) Methodological characteristics of EtR in Chinese EB-CPGs over 11 years. (D) Methodological characteristics of EtR in Chinese CB-CPGs over 11 years. 
A Methodological characteristics of EtR in EB-CPGs versus CB-CPGs

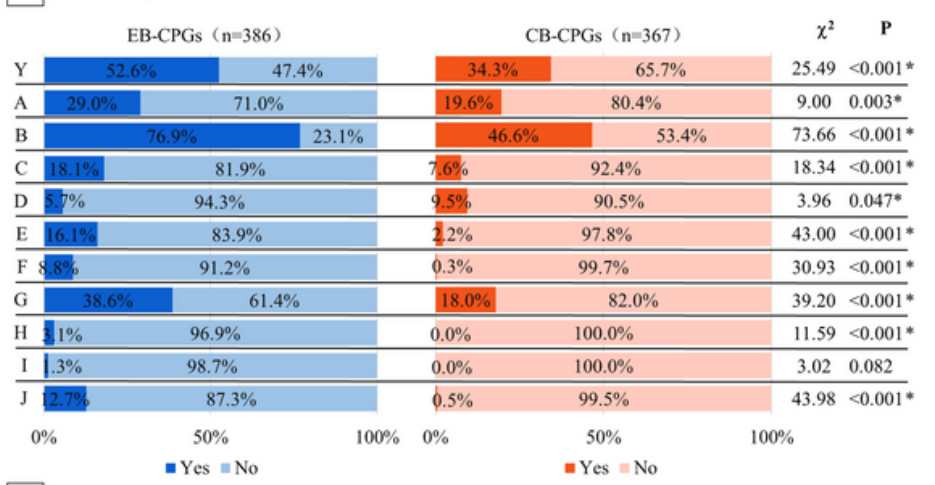

C Multi-factor Binary Logistic regression model for the related factors to the rigor of EtR in Chinese CPGs

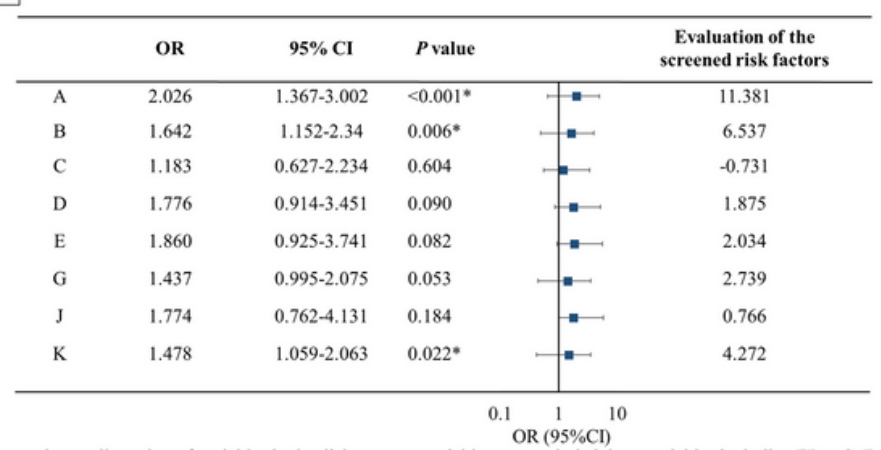

B Methodological characteristics of EtR in CPGs using GRADE versus those not

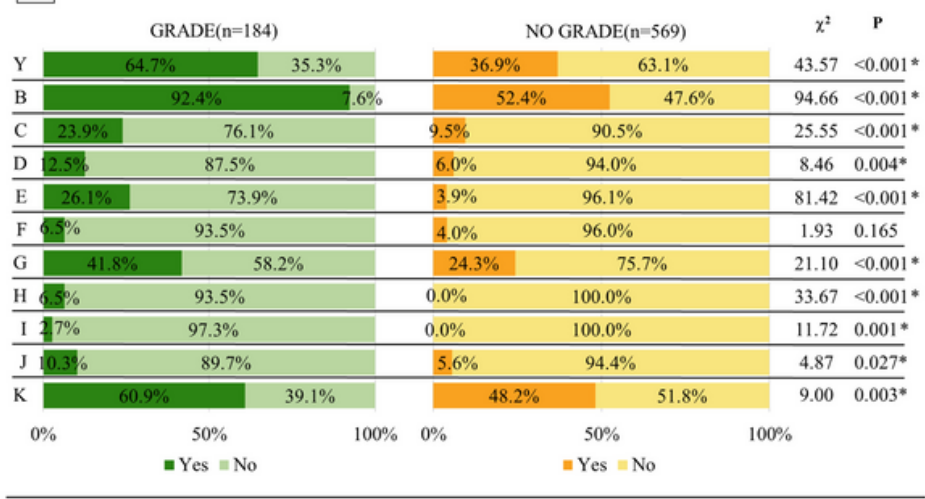

\begin{tabular}{ll}
\hline Y & Considered relevant factors \\
A & GRADE or non-GRADE CPGs \\
B & The level of evidence designated \\
C & The use of the consensus methods \\
D & The holding of consensus meetings to discuss or vote on recommendations \\
E & The participation of methodologists \\
F & The participation of patient \\
G & The declaration of conflicts of interest \\
H & The production of EP or SoF tables \\
I & The use of supporting tools \\
J & The conduct of external reviews \\
K & EB-CPGs or CB-CPGs
\end{tabular}

Due to the small number of variables in the dichotomous variables, we excluded three variables including EP or SoF tables produced, participation of patients, the supporting tools used. $* P<0.05$

\section{Figure 3}

Statistical analysis of the methodological characteristics of the EtR in Chinese CPGs during 2010-2020. (A) Methodological characteristics of EtR in EB-CPGs versus CB-CPGs. (B) Methodological characteristics of EtR in CPGs using GRADE versus those not. (C) Multi-factor Binary Logistic regression model for the related factors to the rigor of EtR in Chinese CPGs.

\section{Supplementary Files}

This is a list of supplementary files associated with this preprint. Click to download.

- Supplementarytable.docx 